\title{
Solar Power Capture using Polymeric Optical Fibres
}

\author{
Kevin Jagadissen Munisami \\ University of West London \\ kevin.munisami@uwl.ac.uk
}

\author{
Demetri Kalymnios \\ London Metropolitan University \\ d.kalymnios@tiscali.co.uk
}

\author{
Bal Virdee \\ London Metropolitan University \\ b.virdee@londonmet.ac.uk
}

\author{
Nicholas Ioannides \\ Dexiotis Ltd \\ nicholas@dexiotis.com
}

\begin{abstract}
The design and performance of a novel low cost technique for solar power capture is presented. Applications for such a system include daylighting, solar power delivery and in electricity generation systems using algae, amongst others. The proposed model uses Polymeric / Plastic Optical Fibres (POF) not only as the light transport medium but also as the light collecting mechanism. The light collecting arrangement is based on the Reciprocity Principle and consists of the curved surface of a POF that is structurally modified so that solar light can be directed into the fibre by using an analogous process to fibre side emission but in the reverse. Several arrangements have been investigated. Computer modeling confirms the achievability of an acceptance cone of at least $\pm 50^{\circ}$. Empirical results show a $53 \%$ increase in efficiency with $3 \mathrm{~mm}$ POF compared to $2 \mathrm{~mm}$ ones. A major advantage of this technique is the elimination of one of the two axes (orbital) of solar tracking and the considerable decrease in importance of the second axis (seasonal), currently a necessity in solar scanning systems.
\end{abstract}

Index Terms-Angular displacement, daylighting, polymeric optical fibre (POF), refractive index, scattering, solar scanning, solar tracking.

\section{INTRODUCTION}

Plastic Optical Fibre (POF) was developed as a cheap alternative to its glass counterpart almost 3 decades ago. However in the last 10 years, it has proven to be a better and cheaper substitute for several applications including short haul communication typically in the range of $100 \mathrm{~m}$ to $1 \mathrm{~km}$ with data rates up to $1 \mathrm{Gbps}$. Local Area Networks and FibreTo-The-Home (FTTH) are examples of such applications. POF has also been extensively used in other applications such as medical sensing, automotive engineering, illumination and solar power delivery.

The concept of integrating optical fibres in solar energy systems dates back to the 1970s [1] with the development of a fused-silica-core fibre that could propagate solar radiation over a $40 \mathrm{~m}$ distance. Cariou et al [2] continued to work along the same area with the transmission of concentrated sunlight over fibres. Incorporating fibres in a three-dimensional Compound Parabolic Concentrator (CPC) was another investigative attempt [3]. Liang et al [4] explored the feasibility of fibre bundles with hexagonal inputs, capable of transmitting $100 \mathrm{~W}$ of concentrated solar energy each and achieved transmission efficiency of $70 \%$.

Fibre optic mini-dishes were also reported [5] with efficiencies reaching $80 \%$ and high level of solar concentration. However, this design utilised mechanical components and customised concentration optics, thus increasing the overall cost. A similar concept was also investigated for high temperature solar receivers [6].

All these attempts at incorporating fibres in solar energy systems were made futile due to their high cost, high attenuation coefficients, their low numerical aperture and the limited types of fibre available in those days [7]. Since then the fibre optic industry has undergone significant technological advancement, leading to the development of fibres with better and more importantly customisable performances at reduced manufacturing costs. The introduction of POF and other types of fibres in the market has renewed the interest in solar applications due to their low cost and also their high numerical apertures, an appropriate condition for lighting applications and solar power transmission. More recently POF has successfully been incorporated in several commercial daylighting systems [8]. However, their uses in such systems have been limited to being the transport medium only and this argument establishes the rationale behind this research.

Therefore, the main aim of this project is to develop an innovative technique to not only collect solar energy but also transport it, by using POF.

\section{Proposed DESIGN}

\section{A. Light Collecting Mechanism}

This paper introduces preliminary work done on a novel design for a light collection mechanism using low cost POF as the light capturing medium as well as the transmitting one. Research so far has shown that no other such system uses POF as both the collection and transport media without the need for concentration optics. The concept adopted in the proposed design is to capture light on the side surface of the fibre, as opposed to the fibre end, as is traditionally the case. By structurally modifying the external surface of the POF, light can be forced and confined into the core by a process comparable to side emission. The 'imperfected' surface enables light rays to penetrate into and propagate along the length of the fibre through total internal reflection (TIR).

The light capturing part of the system, consisting of curved, structurally modified POFs, was designed by considering the sun's trajectory and seasonal variations. Although the intensity of sunlight falling on a surface is mainly based on parameters such as scattering due to clouds, atmospheric dusts and other optically active scattering and diffusion of radiation, yet the angle between the sun and the normal of the object is also of paramount importance [9]. 
The sun's elliptical orbit around the earth causes a variation of $6 \%$ in the amount of annually received solar radiation. This figure is also influenced by the seasonal inclination of $\pm 23.5^{\circ}$ from the perpendicular axis (equator).

The East to West orbit and the seasonal variations of the Earth's position with respect to the sun are also illustrated in Figure 1 as a coordinate system for an observer at point Q. Also displayed are the azimuth angle $(\gamma)$, the altitude angle $(\alpha)$ and the zenith angle $(Z)$ for a central sun ray along direction vector $(\mathrm{S})$. The unit vectors $i, j, k$ along their respective axes are also shown.

The sun's position at any given point in time can therefore be defined from two important parameters:

- The solar azimuth $(\gamma)$ which is the angle between the true south and the point on the horizontal directly below the sun.

and either:

- The solar altitude $(\alpha)$ which is the angle between the horizontal plane and the line to the centre of the sun.

or:

- The solar Zenith angle (z) which is the complement of the altitude angle.

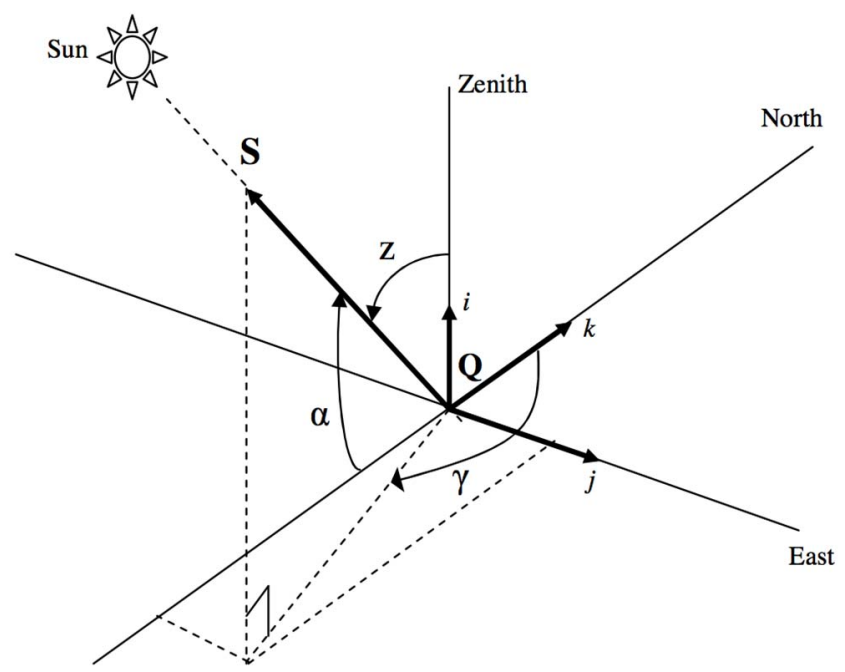

Fig. 1. Illustration of the earth surface coordinate system for an observer at point Q.

The performance of any system using solar energy is directly related to the amount of sunlight acquired, which makes solar tracking in both axes a vital part of such a system [10]. The inherent cylindrical shape of the optical fibre immediately lends itself to an arrangement with the need for one of the solar tracking axis eliminated. In order to make the $2^{\text {nd }}$ sun-tracking axis redundant, it is important to curve the POF axis as shown in Figure 2. In this configuration, irrespective of the sun's position during the solar powercapturing period, the optical axis of the sun rays incident on the POF solar capturing side will always be perpendicular to the tangent of the POF surface. This does not however minimize the need for the initial proper positioning and alignment during installation of the curved POF on the earth with respect to the solar orbital and seasonal variations.
Figure 2 shows that the solar capturing area of the POF will not be used to its entirety, but only the area receiving direct sunlight. This is a minor point and extending this capturing area will not compromise the arrangement.

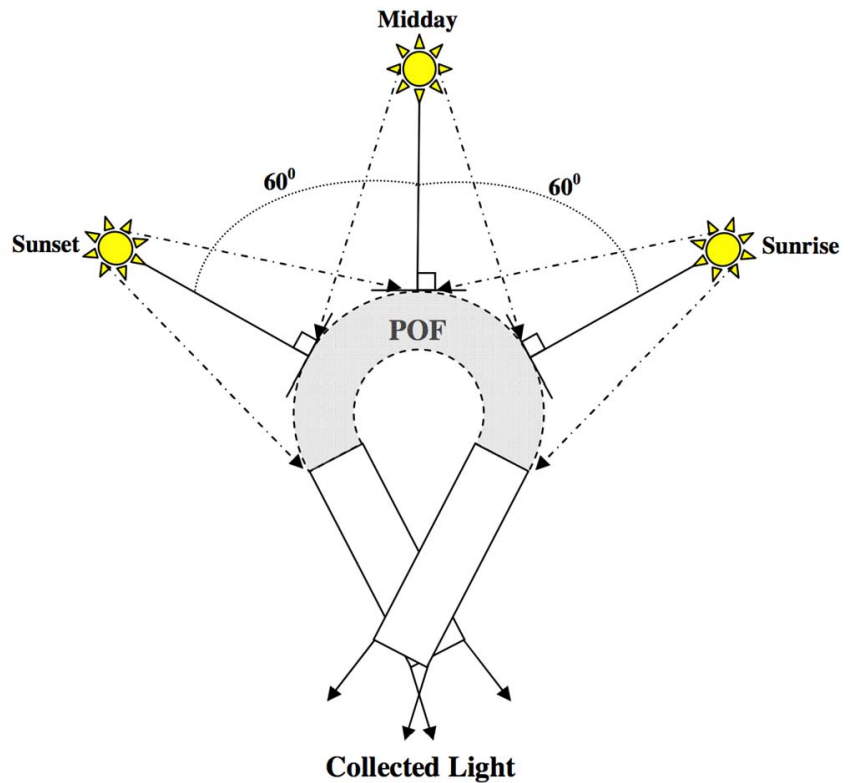

Fig. 2. Illustration of the POF curved arrangement showing the elimination of the second sun tracking axis.

The computer modeling of this configuration, as shown in Figure 3, verifies this hypothesis.

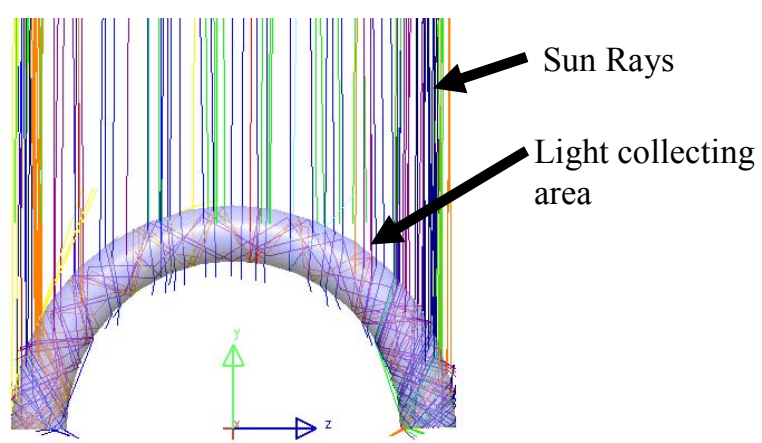

Fig. 3. Computer model to simulate the distribution of light on fibre

The illustration in Figure 3 depicts the curved collecting mechanism over which a number of parallel sunrays are incident. This configuration was modeled for the light source positioned perpendicularly to the Z-axis, with angular displacement of $-50^{\circ}$ to $+50^{\circ}$. This angular range simulated the movement of the sun during the day.

The light distribution incident on the curved POF is given in Figure 4 and the red spread represents the highest order of power. An angular displacement on the left is indicated by a positive angle while a movement on the right is denoted by a negative angle. It can be clearly observed that when the light source is positioned perpendicularly to the POF axis, i.e., at $0^{\circ}$, there is an even distribution of light on the fibre capturing area. 


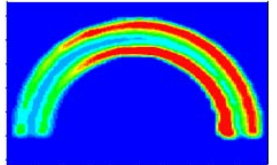

(a) $-50^{\circ}$

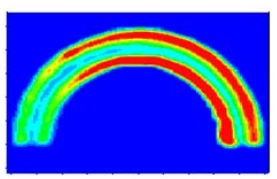

(b) $-40^{\circ}$

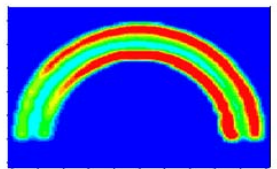

(c) $-30^{\circ}$

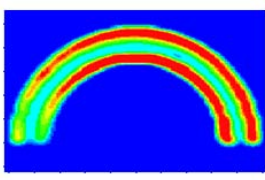

(d) $-20^{\circ}$

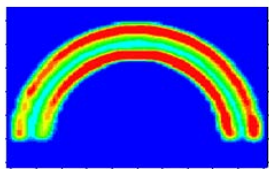

(e) $-10^{\circ}$

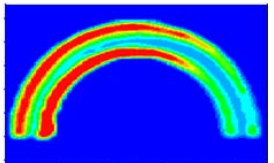

$(\mathrm{g})+50^{\circ}$

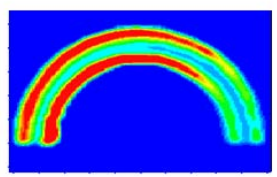

(h) $+40^{\circ}$

(f) $0^{\circ}$

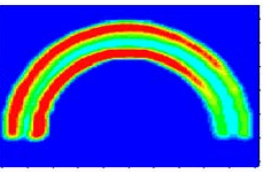

(i) $+30^{\circ}$

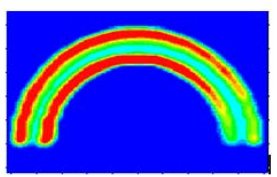

(j) $+20^{\circ}$

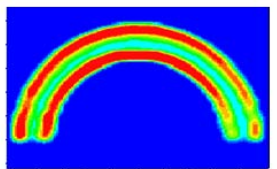

(k) $+10^{\circ}$

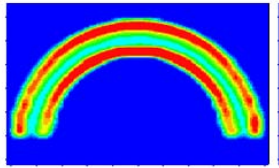

Fig. 4. Distribution of light on fibre at different angles.

It can also be seen (fig. 4) that the distribution of light moves to the side where the source is and the light incident on the other side decreases as the angular displacement increases. As a result, one end of the fibre will output more light than the other. This simple modeling shows that for such a range of angles, there will always be an area of the curved POF facing the sun, thereby confirming the feasibility of this model for eliminating one of the two axes of solar tracking.

\section{B. Quantisation of Captured Light}

The first step in evaluating the effectiveness of this model was considered to be the quantification of the light incident on the surface of the fibre. For a comparative study, a flat surface was first considered followed by a straight POF and a curved POF, for 1, 2 and $3 \mathrm{~mm}$ POF. A sample of area $30 \mathrm{x}$ $(\mathrm{w}=1,2$ or 3$) \mathrm{mm}^{2}$ was used, broken down into $10 \mu \mathrm{m} \mathrm{x}$ $10 \mu \mathrm{m}\left(100 \mu \mathrm{m}^{2}\right)$ units (cells) and the amount of light incident on the surface was referenced with a Q-point (figures 5, 6). The amount of light incident on each cell in each approach was determined using solid angle effects. The results obtained from flat surface and straight POF for all three POF were almost identical. However, a difference of $1 \%$ is observed for $1 \mathrm{~mm}$ POF, $0.5 \%$ for $2 \mathrm{~mm}$ POF and $0.35 \%$ for $3 \mathrm{~mm}$ POF when comparing with the light levels of a flat surface.

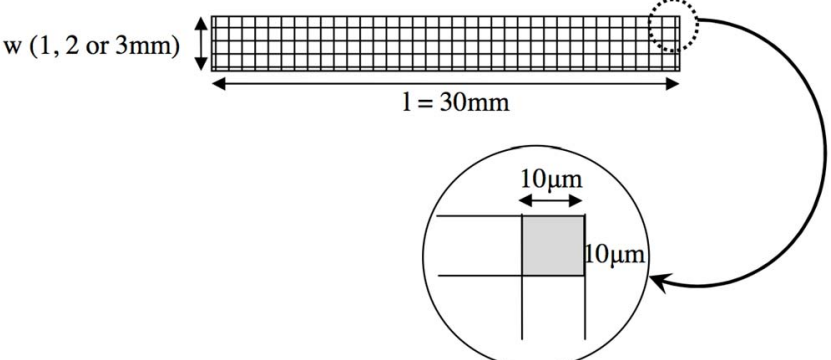

Fig. 5. Illustration of a 'cell' arrangement considered in the analysis

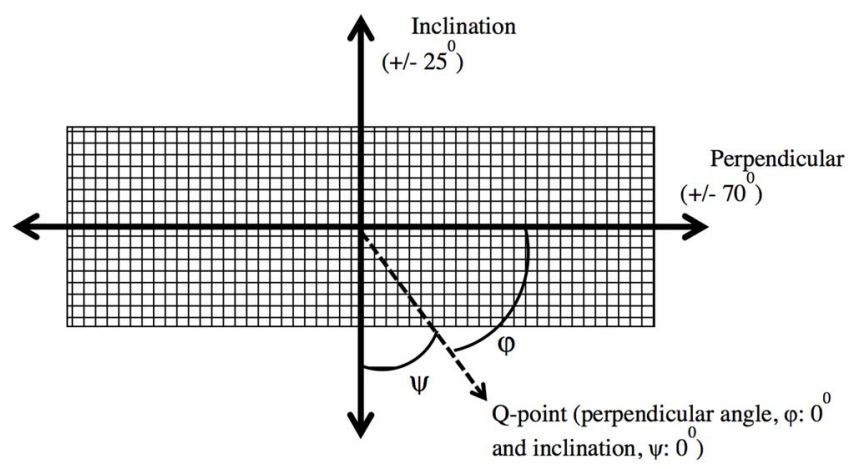

Fig. 6. Solid angle effects as considered in the analysis

The larger surface area of the curved POF as compared to the other sample, due to its characteristic cylindrical shape and the introduced curvature, inherently comprised a larger number of unit cells. Taking into account the introduction of a curvature of radius $15 \mathrm{~mm}$ to the POF the parameters for seasonal and orbital variations are shown in Tables I and II.

TABLE I

POF PARAMETERS FOR SEASONAL VARIATION

\begin{tabular}{lccc}
\hline & $\mathbf{1 m m}$ POF & $\mathbf{2 m m}$ POF & $\mathbf{3 m m}$ POF \\
\hline Radius & $0.5 \mathrm{~mm}$ & $1 \mathrm{~mm}$ & $1.5 \mathrm{~mm}$ \\
\hline Perimeter & $3.142 \mathrm{~mm}$ & $6.283 \mathrm{~mm}$ & $9.425 \mathrm{~mm}$ \\
\hline Perimeter / & $1.571 \mathrm{~mm}$ & $3.142 \mathrm{~mm}$ & $4.712 \mathrm{~mm}$ \\
\hline Cells on strip & 157 & 315 & 471 \\
\hline $\begin{array}{l}\text { Angular step from } \\
\text { Q-point (deg) }\end{array}$ & 1.145916 & 0.572958 & 0.381972 \\
\hline
\end{tabular}

TABLE II

POF PARAMETERS FOR ORBITAL VARIATION

\begin{tabular}{lccc}
\hline & $\mathbf{1 m m}$ POF & 2 mm POF & 3mm POF \\
\hline Radius & $15 \mathrm{~mm}$ & $15 \mathrm{~mm}$ & $15 \mathrm{~mm}$ \\
\hline Perimeter & $94.25 \mathrm{~mm}$ & $94.25 \mathrm{~mm}$ & $94.25 \mathrm{~mm}$ \\
\hline Perimeter / & $47.125 \mathrm{~mm}$ & $47.125 \mathrm{~mm}$ & $47.125 \mathrm{~mm}$ \\
\hline Cells on strip & 4712.5 & 4712.5 & 4712.5 \\
\hline $\begin{array}{l}\text { Angular step } \\
\text { from Q-point } \\
\text { (deg) }\end{array}$ & 0.038197 & 0.038197 & 0.038197 \\
\hline
\end{tabular}


The increased number of light cells incorporated in the new surface area is shown in table III.

TABLE III

DIMENSIONS AND NUMBER OF CELLS ON CURVED POF

\begin{tabular}{cc}
\hline Curved POF Surface Area & Number of $\mathbf{1 0 0} \boldsymbol{\mu m}^{2}$ cells \\
\hline $\begin{array}{c}47.125 \mathrm{~mm} \times 1.571 \mathrm{~mm}= \\
74.0 \mathrm{~mm}^{2}\end{array}$ & $4712.5 \times 157.1=740,334$ \\
\hline $\begin{array}{c}47.125 \mathrm{~mm} \times 3.142 \mathrm{~mm}= \\
148.1 \mathrm{~mm}^{2}\end{array}$ & $4712.5 \times 314.2=1,480,668$ \\
\hline $\begin{array}{c}47.125 \mathrm{~mm} \times 4.712 \mathrm{~mm}= \\
222.1 \mathrm{~mm}^{2}\end{array}$ & $4712.5 \times 471.2=2,220,530$ \\
\hline
\end{tabular}

The seasonal variations, characterised by an angular displacement along the cylindrical surface, for the $3 \mathrm{~mm}$ POF are depicted in Figure 7. As expected, maximum light levels occurs with the sun positioned perpendicularly and with no inclination at the Q-point. Introducing angular displacements shows a decrease in light level as well as a shift of the arch towards the negative or positive part of the graph accordingly. The maximum light level collected would however be at the point representing the displacement.

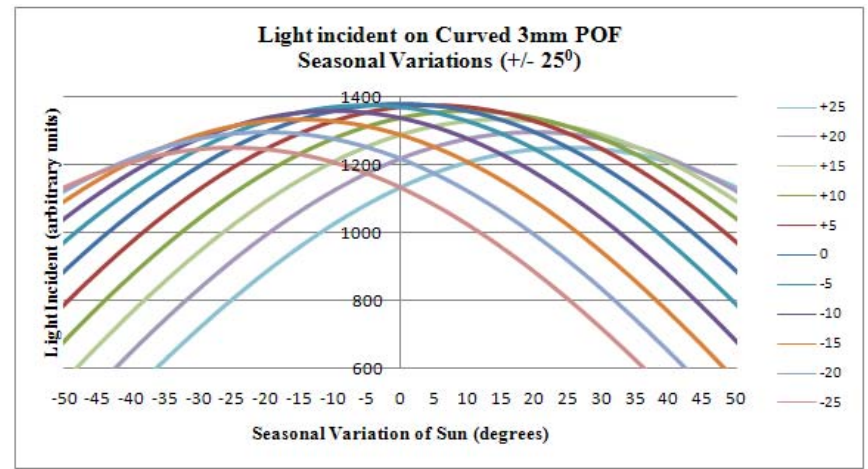

Fig. 7. Light incident on POF w.r.t angle of incidence for seasonal variations

The orbital variations on the other hand, as shown in figure 8 , are represented by angular displacements along the introduced curvature of the POF. The maximum light level as anticipated occur at the same point as the previous case.

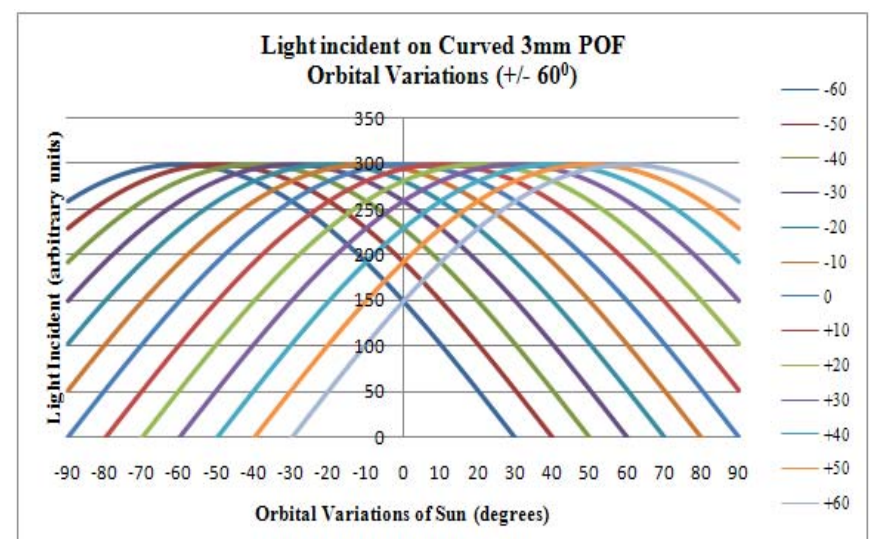

Fig. 8. Light incident on POF w.r.t angle of incidence for orbital variations
As angular displacements are introduced, a similar shift in the arch is observed. However the light level remains constant as opposed to the ones for the seasonal variations. This clearly indicates the elimination of the orbital tracking axis, hence confirming the validity of the hypothesis based on the curved POF arrangement.

\section{Structural Modifications}

The smooth surface of the optical fibre renders it difficult to scatter light within the core of the POF to act as collection medium. Taking into account the refractive indices of the core $\left(n_{1}=1.49\right)$ and cladding $\left(n_{2}=1.40\right)$, the critical angle, $\mathrm{Q}_{\mathrm{c}}$, at the core-cladding interface is calculated using Snell's law [11], to be $70^{\circ}$. As a result, for TIR to occur a minimum incident angle of $89^{\circ}$ is necessary. Thus rays hitting the POF surface at lower angles will not be confined within the core but will instead be refracted out at the core/cladding boundary, hence making this arrangement a highly inefficient light capturing mechanism. Since the refractive indices of the POF materials cannot be altered to yield a lower $Q_{c}$, a methodology was adopted to increase the refracted angles of the rays entering the core. This was achieved through scattering the incident light at the surface of the POF by modifying the structure of the POF surface. Controlled roughening of the light capturing area scatters the light at an angle larger than the critical angle, therefore decreasing the amount of light refracted out and confining more light into the core for propagation down the POF by TIR. For comparison, magnified images of smooth and rough surface of a POF, taken with a scanning electron microscope, are shown in Figure 9.
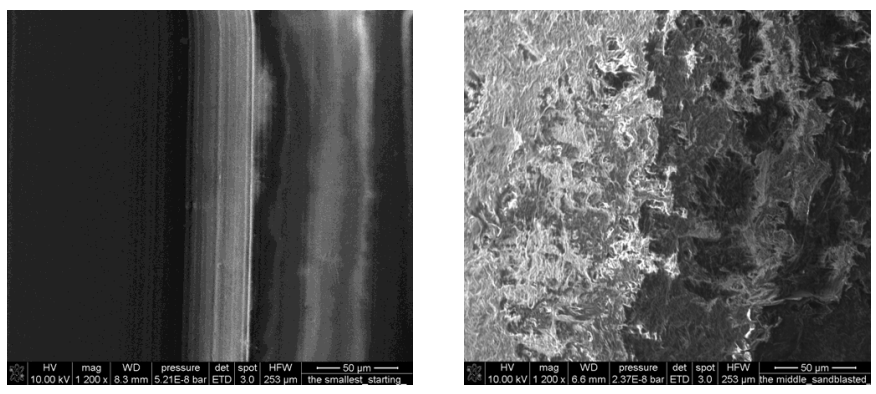

Fig. 9. POF smooth and "imperfected" surfaces at $1200 \mathrm{X}$

The depth of the roughness is in the micrometer range and extremely random. This makes the analysis of structurally modified POF very complex.

Rayleigh and Mie scattering are the most commonly used theories for investigating such structures [12]. However, since the roughness of the surface can be considered as scatterers with sizes larger than the nanometer-scaled wavelengths of visible light, Mie theory is preferred. Among the assumptions made for this analysis is the one that the roughness is considered as tiny spheres on the surface of the POF and that they have the same refractive index. It is also assumed that scattering of one sphere can be integrated to yield the behaviour of larger areas. 
One way of approaching this analysis is to consider the solid angle defined from the rate of scattered energy due to a single spherical scatterer, as illustrated in Figure 10.

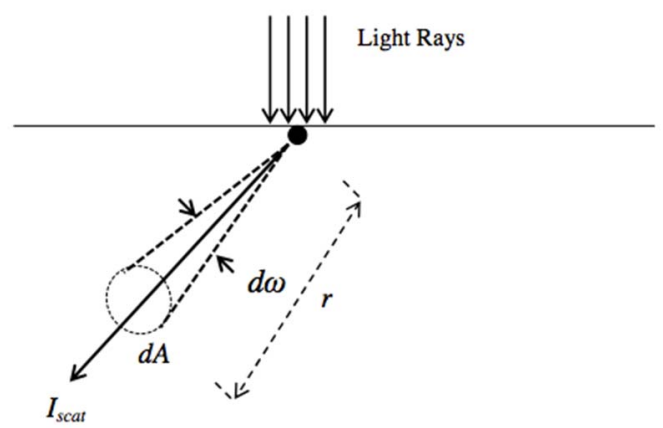

Fig. 10. Energy scattering resulting from a single sphere

The total scattered energy incident on $d A$ is derived using differential scattering cross section and is given as [12]:

$E_{\text {scat }}=I_{0} \sigma_{\text {scat }} d \omega$

where $\sigma_{\text {scat }}$ is the average of the vertically and horizontally polarised cross sections, and:

$\sigma_{\text {scat }}=\frac{d A}{r^{2}}$

This can be further simplified to give the redistribution of light from scattering as:

$I_{\text {scat }}=I_{0} \frac{1}{r^{2}} \sigma_{\text {scat }}$

For unpolarised incident light, the differential cross section derived from (3), yields:

$\sigma_{\text {scat }}=\frac{\lambda^{2}}{8 \pi^{2}}\left(i_{1}+i_{2}\right)$

where the angular intensity parameters $i_{1}$ and $i_{2}$ are defined as [13]:

$i_{1}=\left|\sum_{n=1}^{\infty} \frac{2 n+1}{n(n+1)}\left[a_{n} \pi_{n}(\cos \theta)+b_{n} \tau_{n}(\cos \theta)\right]\right|^{2}$

$i_{2}=\left|\sum_{n=1}^{\infty} \frac{2 n+1}{n(n+1)}\left[a_{n} \tau_{n}(\cos \theta)+b_{n} \pi_{n}(\cos \theta)\right]\right|^{2}$

The main conclusion based on the derived expressions is that the distribution of the scattered light is dependent on the intensity of the incident light, the dimension and material characteristic properties of the scatterers. The scattered distribution is also dependent on the scattering angle.

The scattering distribution due to the roughness on the POF surface was also empirically investigated. Figures 11 and 12 show intensity of a green HeNe laser emitting at 532 $\mathrm{nm}$ when scattered through a smooth surface and a structurally modified one respectively.

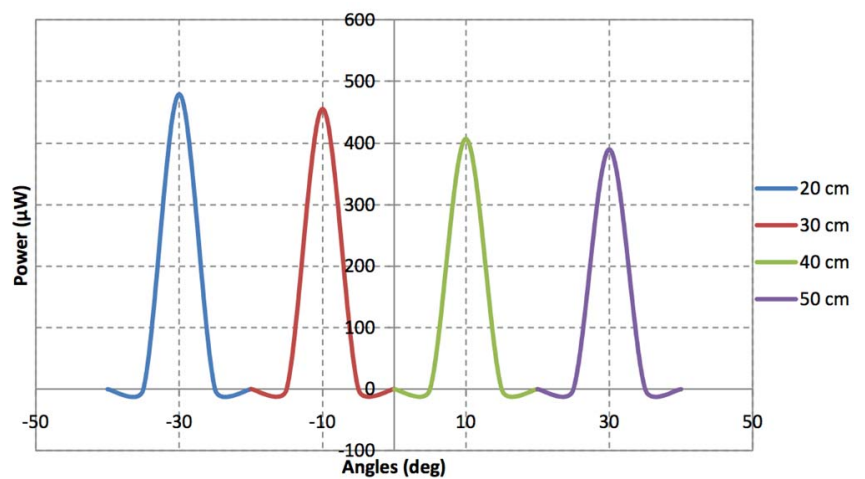

Fig. 11. Intensity of green laser output at various distances through smooth surface.

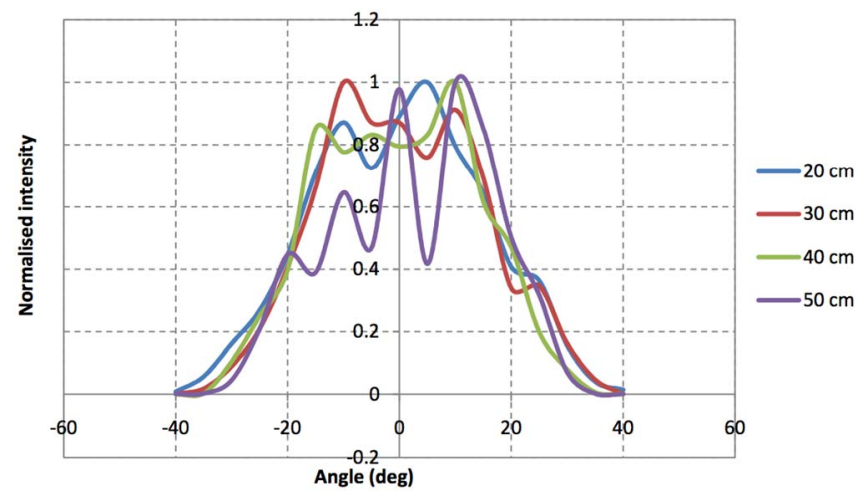

Fig. 12. Normalised distribution of light scattered through a roughened surface.

A very narrow spectrum is observed for the smooth surface and the full width half maximum (FWHM) angle is averaged to approximately $4^{\circ}$. This value increases to $40^{\circ}$ for the structurally modified surface, as shown in the normalised distributions in Figure 11. This indeed confirms that roughening the surface of $\mathrm{POF}$ is a feasible approach to create a scattering optical surface. The irregular shape of the waveforms is indicative of the randomness of the scattering surface, which nevertheless produces roughly the same broadening of the FWHM angles.

\section{CONCLUSION}

A novel approach for solar power capture using POF as the collection and transport mediums has been investigated. This method uses a process analogous to fibre side emission but in the reverse. Due to the fact that a smooth surface will simply refract light out of the fibre, the surface of the POF is structurally modified to allow light to be scattered and confined in the core. Introducing a $30 \mathrm{~mm}$ diameter curvature further improves the light capturing efficiency.

Analytical investigations prove the validity of the hypothesis that a curved POF surface is a better light collector than a straight one. Angular displacements due to seasonal variations cause a shift of the maximum intensity point as well as a decrease in intensity with a move away from the perpendicular point. The same shift is observed for orbital angular displacement but without the drop in intensity, thus showing the elimination for the need for solar tracking. 
Theoretical analysis and experimental results verify the importance of introducing roughness on the curved surface for improved performance.

The next step in this research would be to investigate the efficiency of the system with a view to optimise its performance. This could be tackled by creating a prototype and perform optical measurements based on the input power density and power output. A bidirectional scattering distribution function measurement would also be useful in characterising the structural modification of the POF surface in order to optimise the light capture.

\section{REFERENCES}

[1] D. Kato and T. Nakamura, "Applications of Optical fibers to the transmission of solar radiation,.” J. Applied Physics, 47, pp.4528-4531, 1976.

[2] J.M. Cariou, L. Martin, J. Dugas "Concentrated solar energy transport by optical fibres, physics of fibre optics," Adv Ceramics, Vol. 2, 1981.

[3] N. Khatri, M. brown and F. Gerner, "Using fiber optics to tap the sun's power," Int. Comm.in heat and mass transfer, volume 20, issue $6 \mathrm{pp}$. 771-781, 1993.

[4] D. Liang, Y. Nunes, L. Monteiro and M. Collares-Peirera, "200W solar energy delivery with optical fiber bundles," Nonimmaging Optics: Maximum Efficiency Light Transfer IV, Vol. 3139, SPIE, Bellingham, Washington, 1997.

[5] D. Feuermanna, J. M. Gordonab, "Solar fiber-optic mini-dishes: a new approach to the efficient collection of sunlight," Solar Energy, Vol. 65, Issue 3 pp.159-170, 1999.

[6] J Karni, A Kribus, R Rubin, D Sagie, P Doron, A Fiterman, "The DIAPR: a high pressure, high temperature solar receiver," J. Solar Energy Eng., 119 p. 74, 1997.

[7] A. Kribus, O. Zik, J. Karni, "Optical fibers and solar power generation", Solar Energy, 5 pp. 405-416, 2000.

[8] J. Munisami and D. Kalymnios, "Novel technique for solar power illumination using plastic optical fibres, Proc. SPIE, Vol. 7103 71030D, 2008.

[9] Lee et al, "Sun Tracking Systems: A Review," Sensors, 9, 3875-3890, 2009.

[10] Mousazadeh et al, "A review of principle and sun-tracking methods for maximizing solar systems output", Renewable and Sustainable Energy Reviews 13, pp. 1800-1818, 2009.

[11] A. W Synder, and J.D Love, Optical Waveguide Theory, Wilshire, Antony Rowe Ltd, 1983.

[12] C. F. Bohren and D. R. Huffman, “Absorption and scattering of light by small particles”, Wiley, New York, 1998.

[13] M. L. Mishchenko, L. D. Travis, and A. A. Lacis, "Scattering, Absorption, and Emission of Light by Small Particles", Cambridge University Press, Cambridge, 2002. 